\title{
Analisando o cenário brasileiro de pesquisa de objetos de aprendizagem.
}

\author{
João Pedro Dewes Guterres ${ }^{1}$ e Milene Selbach Silveira ${ }^{1}$ \\ ${ }^{1}$ Faculdade de Informática, Programa de Pós-Graduação em Ciência da Computação \\ Pontifícia Universidade Católica do Rio Grande do Sul (PUCRS) - Porto Alegre, RS - \\ Brasil \\ joao.guterres@acad.pucrs.br, milene.silveira@pucrs.br
}

\begin{abstract}
In this paper we present an overview of the Brazilian research about learning objects, considering the analysis of publications in conferences related to the Brazilian Computer Society's Council for Computers in Education. We performed a systematic mapping about these conferences' publications of the last 15 years - from the date on which the term "learning object" spread, and the research on learning objects began to be published in Brazil. From the analysis of 205 publications, we present the main research areas in this field as well its major research initiatives.
\end{abstract}

Resumo. Neste artigo apresentamos um panorama sobre a pesquisa brasileira em objetos de aprendizagem, a partir da análise de publicações efetuadas nos principais eventos relacionados à Comissão Especial de Informática na Educação da Sociedade Brasileira de Computação. Para isso, realizamos um mapeamento sistemático sobre as publicações dos últimos 15 anos destes eventos - a partir da data na qual o termo objeto de aprendizagem se difundiu e as pesquisas sobre objetos de aprendizagem começaram a ser publicadas. A partir da análise de 205 publicações, são apresentadas as principais vertentes e iniciativas de pesquisa sobre o tema no país.

\section{Introdução}

Com a concepção do termo "objeto de aprendizagem" no início dos anos 2000 [Wiley 2000, Gibbons 2000] aliada aos avanços tecnológicos, a produção desses recursos por meio de inciativas individuais ou de laboratórios institucionais, vem se tornando cada vez mais comum. Existem muitas as definições para o termo objetos de aprendizagem, algumas mais abrangentes e outras bem específicas sobre o tema. Com isso, decidiu-se adotar a definição de que aborda de forma abrangente as principais características dos objetos: "quaisquer materiais eletrônicos (como imagens, vídeos, páginas web, animações ou simulações), desde que tragam informações destinadas à construção do conhecimento (conteúdo autocontido), explicitem seus objetivos pedagógicos e estejam estruturados de tal forma que possam ser reutilizados e recombinados com outros objetos de aprendizagem (padronização)" [Carneiro e Silveira 2014].

$\mathrm{O}$ uso dos objetos de aprendizagem tem demonstrado ser eficiente no aperfeiçoamento do processo de ensino e aprendizagem [Damasceno et al. 2014], de modo a promover autonomia no processo de aprendizagem [Bocchese et al. 2013] permitindo ao aluno ser o sujeito ativo de sua aprendizagem [Pinho e Eliasquevici 
V Congresso Brasileiro de Informática na Educação (CBIE 2016)

Anais do XXVII Simpósio Brasileiro de Informática na Educação (SBIE 2016)

2008]. Sendo assim, considerando a importância desse assunto para a área de informática na educação, a comunidade brasileira começou a investigar o tema e logo publicações sobre pesquisas e produções de objetos começaram a fazer parte dos eventos relacionados à Comissão Especial de Informática na Educação (CE-IE) da Sociedade Brasileira de Computação (SBC).

Além disto, de modo a fomentar a produção de objetos de aprendizagem no Brasil, em 2000 o Governo Federal por meio do Ministério de Educação e Cultura lançou o Projeto RIVED com o propósito de desenvolver módulos educacionais digitais [Cintra 2010]. Em 2004, iniciou-se o processo de transferência de produção de atividades educacionais digitais e interativas em forma de objetos de aprendizagem, por meio do edital "Projeto RIVED/Fábrica Virtual". Com isso, foram selecionadas Instituições Públicas Brasileiras de Ensino Superior, de modo a promover de um trabalho colaborativo e interdisciplinar de produção desses objetos dentro da academia e não mais pelas Secretarias de Educação.

Coincidentemente, as primeiras publicações encontradas datam de 2004, versando sobre os seguintes assuntos: ontologia para pesquisa de objetos de aprendizagem baseada na web semântica [Araújo e Ferreira 2004]; integração de objetos de aprendizagem com um ambiente telemático [Macêdo et al. 2004]; abordagem de objetos de aprendizagem com agentes inteligentes [Gomes et al. 2004]; sistema multiagente para compartilhamento de objetos [Brito et al. 2004]; e recuperação paralela de matemática baseado em objetos de aprendizagem [Moura et al. 2004]. Os artigos referenciados, em sua grande maioria, apresentam os objetos de aprendizagem associados a tecnologias emergentes para época, como inteligência artificial, web semântica e novos recursos da web 2.0, popularizada no referido ano da publicação dos artigos.

De modo a explorar o avanço das pesquisas brasileiras sobre o assunto, essa pesquisa busca a identificação das principais vertentes de pesquisa de objetos de aprendizagem. O restante do artigo está organizado da seguinte forma: a seção 2 introduz a metodologia de pesquisa aplicada na pesquisa aqui apresentada, descrevendo a busca por artigos e a categorização realizada; na seção 3 é apresentada uma análise geral dos resultados encontrados e a seção 4 especializa estes resultados, apresentando vertentes e iniciativas de pesquisa sobre o assunto; por fim, a seção 5 apresenta as considerações finais, além de suas limitações, contribuições e trabalhos futuros a partir deste trabalho.

\section{Metodologia}

A fim de se atingir o objetivo deste trabalho - apresentar um panorama da pesquisa sobre objetos de aprendizagem, com foco nas vertentes e iniciativas de pesquisa sobre o tema - inicialmente, realizou-se uma pesquisa nas bases de dados relacionadas à Comissão Especial de Informática na Educação (http://ceie-br.org/), a fim de identificar a existência de mapeamentos sistemáticos sobre pesquisas e produções de objetos de aprendizagem no Brasil. No entanto, nenhum artigo específico foi encontrado sobre o assunto. 
V Congresso Brasileiro de Informática na Educação (CBIE 2016)

Anais do XXVII Simpósio Brasileiro de Informática na Educação (SBIE 2016)

Desta forma, idealizou-se um mapeamento sistemático sobre o tema, considerando as principais conferências brasileiras sobre informática na educação, relacionadas à CE-IE.

Para obtenção das publicações sobre objetos de aprendizagem, foi realizada a busca de artigos completos e resumidos, que estão disponibilizados digitalmente na biblioteca digital da CE-IE. Os eventos selecionados foram o SBIE (Simpósio Brasileiro de Informática na Educação), WIE (Workshop de Informática na Escola), dos Workshops relacionados ao CBIE (Congresso Brasileiro de Informática na Educação) e o DesafIE (Workshop de Desafios da Computação Aplicada à Educação). Destaca-se que os workshops foram incluídos por estarem no contexto do CBIE, que atualmente agrega o SBIE e WIE e o DesafIE. Além disso, nesta etapa da pesquisa, o foco foi direcionado apenas aos eventos, que se acredita ser possível traçar o panorama brasileiro de pesquisa na área.

Devido à indisponibilidade digital dos anais de todas as edições do WIE e dos Workshops do CBIE, não foi possível obter um recorte preciso destes eventos, de modo a realizar uma exploração completa do assunto. Com isso, as publicações obtidas foram as das últimas quinze edições do SBIE (de 2001 até 2015), últimas doze edições do WIE (de 2004 até 2015) e últimas quatro edições dos Workshops do CBIE (2012 até 2015) e somente a edição de 2012 do DesafIE.

A terminologia para denominação de objetos de aprendizagem é muito heterogênea, sendo que alguns autores utilizam, como sinônimos de objetos, termos como construtos digitais de aprendizagem, recursos educacionais e softwares educativos, dentre outros, que também podem ter outros significados, além do que é compreendido como objeto de aprendizagem segundo a definição de Carneiro e Silveira (2014). Neste contexto, para identificarmos as publicações sobre assunto, foi acordada a inclusão de artigos que citam especificamente o termo "objeto de aprendizagem".

Com esta definição, foi pesquisado na base digital da CE-IE, primeiro, se o título e resumo do artigo continham a palavra "objeto". Após foi feita uma filtragem manual por "objetos de aprendizagem" e suas variações. Essa estratégia foi utilizada para filtrar possíveis modificações deste termo, como por exemplo: "objetos inteligentes de aprendizagem", "objetos digitais de aprendizagem", “objetos virtuais de aprendizagem", etc.

Ao total, foram identificadas 205 publicações relacionadas a objetos de aprendizagem entre os anos de 2004 a 2015. A Figura 1 ilustra o percentual de artigos por evento. Pode-se observar que a característica do SBIE de tratar de pesquisa em informática na educação se reflete em uma maior representatividade de artigos neste evento, se comparada, por exemplo, com o WIE, que foca em aplicação na Escola. A menor quantidade nos workshops em geral, se dá tanto pela especificidade dos mesmos quanto pelo número reduzido de edições e de artigos disponíveis digitalmente. Por contar com apenas um artigo analisado, o percentual de artigos do DesafIE consta como menos de $1 \%$ do total. 


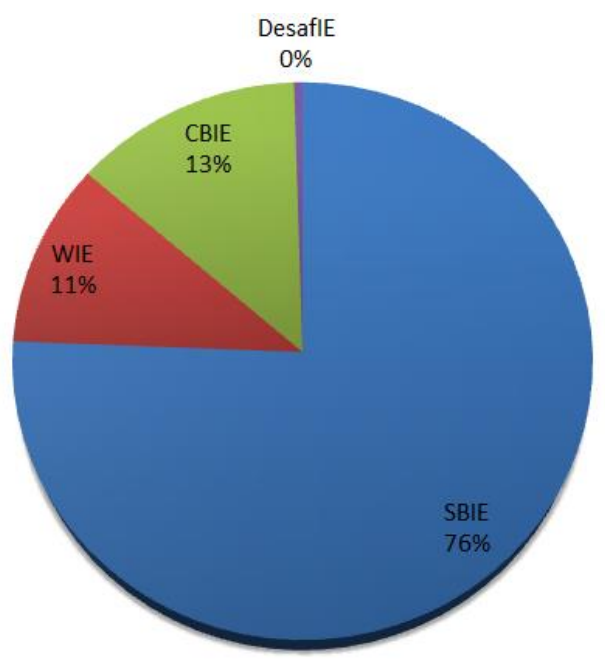

Figura 1. Percentual de artigos por evento.

Para organização e categorização das publicações, as informações de cada artigo foram catalogadas da seguinte forma: (a) Evento; (b) Ano; (c) Título; (d) Autores; (e) Universidades dos autores; (f) Departamento dos autores; (g) Estados dos autores; (h) Categoria da publicação. Devido a pouca representatividade de publicações contendo palavras-chave, essa categoria que inicialmente foi implementada, acabou excluída da análise final.

Após a etapa de cadastramento das informações das publicações, realizou-se o processo de categorização, ou codificação delas. Foi utilizada a estratégia de codificação emergente, citada por Creswell (2013), de modo que criação de códigos, ou categorias, emerge da base de dados. Neste caso, as informações emergiram a partir de observações realizadas após a leitura do resumo e das seções de introdução e conclusão dos artigos. Em caso de dúvida sobre a categorização, realizou-se uma leitura completa do artigo. Todo este processo de coleta e categorização foi realizado de forma manual.

Essa categorização inicial passou por um processo de refinamento, dando origem a versão final das categorias. De modo a padronizar as informações, os dados dos autores, departamentos e universidades foram separados individualmente em uma nova tabela.

Para análise e interpretação dos resultados foram utilizadas tabelas duplicadas quando necessário, de modo a individualizar informações, de acordo com as especificações de cada metadado. Essa pesquisa foi realizada pesquisador pleno com apoio de um pesquisador sênior da área.

É importante ressaltar que devido a manipulação manual dos dados e leitura dos artigos sendo executada por humanos, não se tem a pretensão de afirmar que nossa base e análise possua $100 \%$ de precisão, embora se tenha tido o cuidado de verificar e revisar os dados cadastrados e a análise dos referidos dados.

\section{Análise Geral}

A primeira análise foi realizada a partir da distribuição das publicações durante os anos que foram incluídos na pesquisa. A Figura 2 mostra a quantidade de artigos por ano. Observa-se que o primeiro aparecimento do termo "objeto de aprendizagem" em 
V Congresso Brasileiro de Informática na Educação (CBIE 2016)

Anais do XXVII Simpósio Brasileiro de Informática na Educação (SBIE 2016)

publicações do SBIE surgiu no ano de 2004, aproximadamente 3 anos após o termo se difundir mundialmente. Percebe-se um rápido crescimento de publicações até o ano de 2007 e uma estabilização do número a partir de 2010.

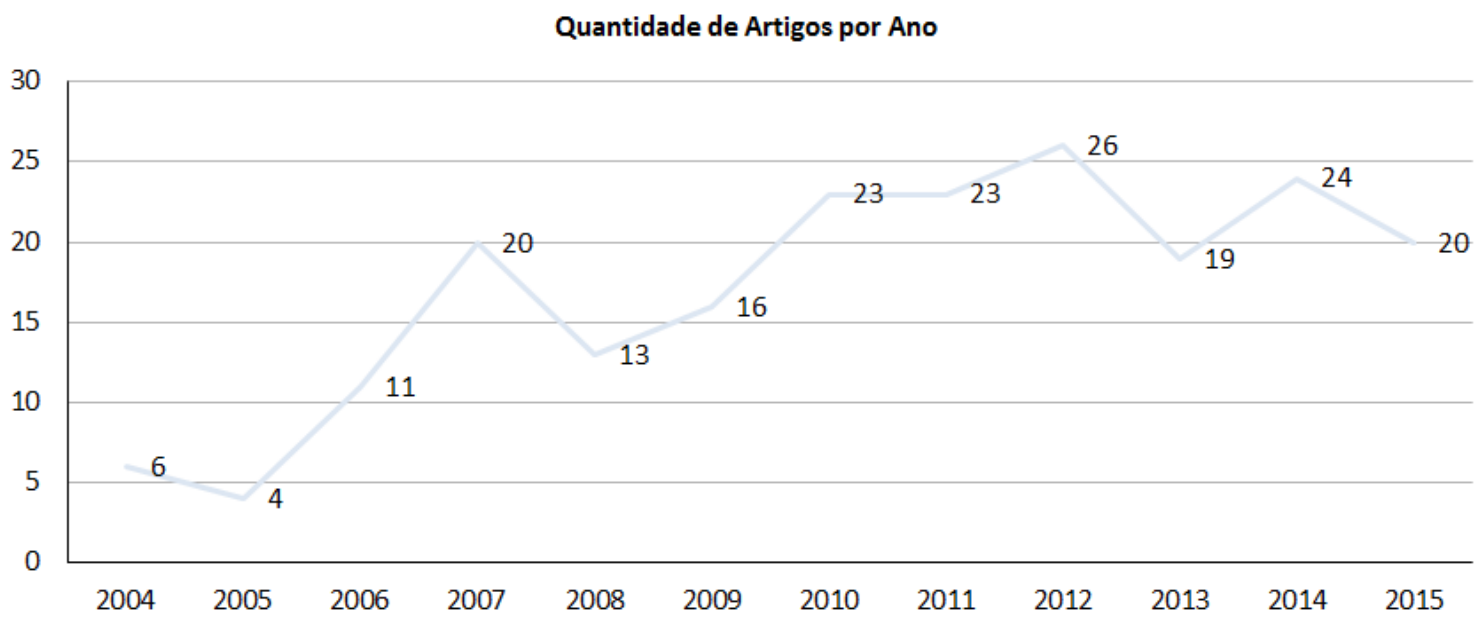

Figura 2. Quantidade de artigos por ano.

A segunda análise foi realizada de modo a verificar os principais autores com publicações referentes a objetos de aprendizagem. Foram identificados 462 autores distintos, contendo uma variância de um a dezenove na quantidade de artigos por autor. A Figura 3 apresenta uma nuvem de tags gerada a partir desta análise, levando em conta os autores e a sua quantidade de publicações cadastradas. A relação do tamanho do nome do autor está representada de acordo com a quantidade de publicações.

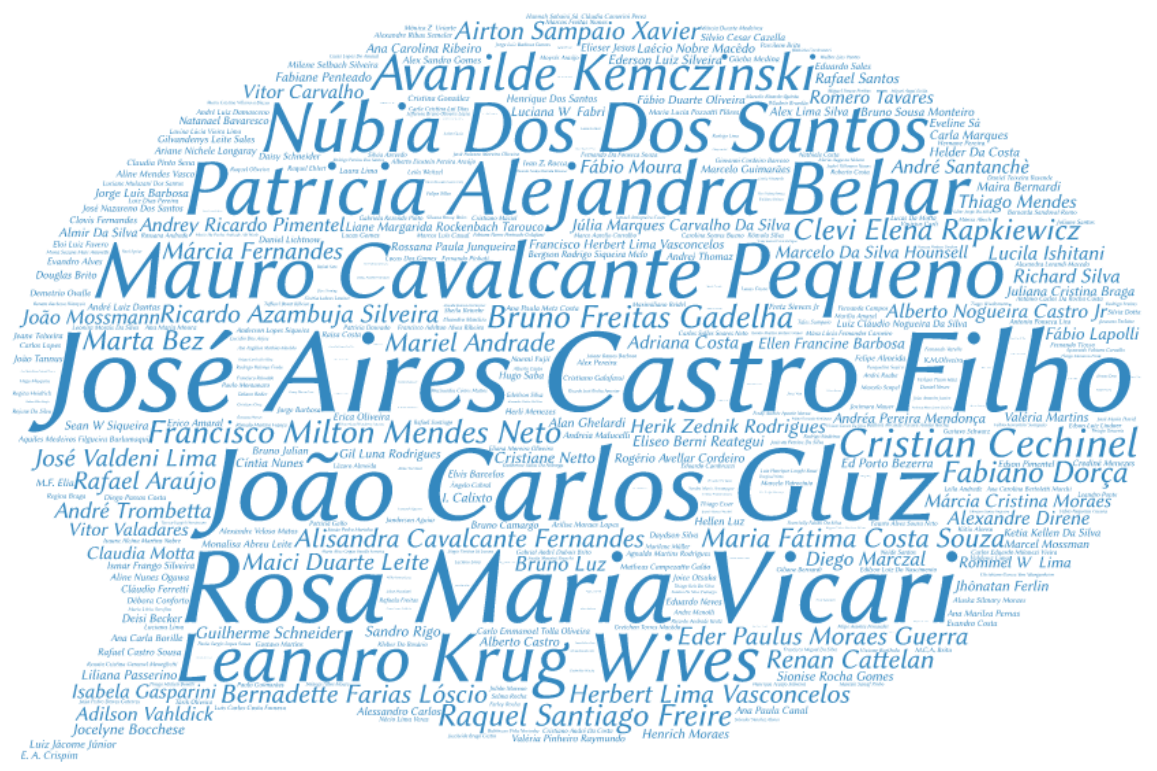

Figura 3. Nome dos principais autores por quantidade de publicação nos eventos pesquisados.

Analisando os departamentos envolvidos nas publicações, evidencia-se a grande heterogenia de áreas envolvidas em pesquisas com objetos de aprendizagem. Contudo, nota-se a representatividade significativa da área da computação, consequência direta de estes serem eventos promovidos pela SBC. A Figura 4 ilustra essa análise de 
V Congresso Brasileiro de Informática na Educação (CBIE 2016)

Anais do XXVII Simpósio Brasileiro de Informática na Educação (SBIE 2016)

departamentos. Os departamentos citados como "Outros" na categorização foram os seguintes: Administração, Aeronáutica, Artes, Ciências, Ciências Aplicadas e Educação, Ciências Aplicadas e Sociais, Ciências Aplicadas e Sociais, Comunicação e Artes, Exatas, Gestão Estratégica de Vendas e Marketing de Relacionamento, Inteligência Aplicada, Tecnologia da Informação e Comunicação, Tecnologia da Informação e Comunicação.
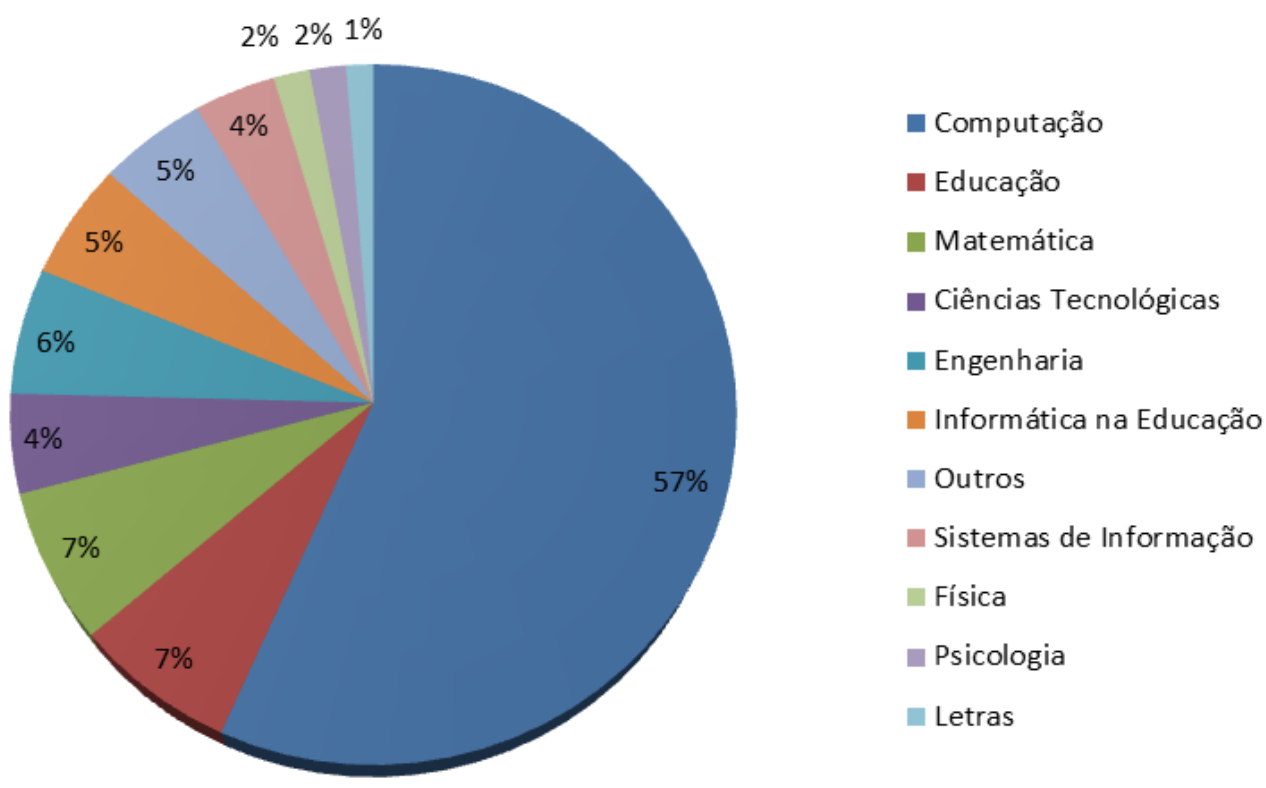

Figura 4. Quantidade de artigos por departamento.

De modo a analisar as principais instituições com publicações referentes a objetos de aprendizagem, nossa quarta análise foi realizada com a relação de instituições relativas aos autores. Foram identificadas 102 Universidades distintas, contendo uma variância de um a 35 na quantidade de artigos por Universidade. A Figura 5 apresenta uma nuvem de tags gerada a partir desta análise.

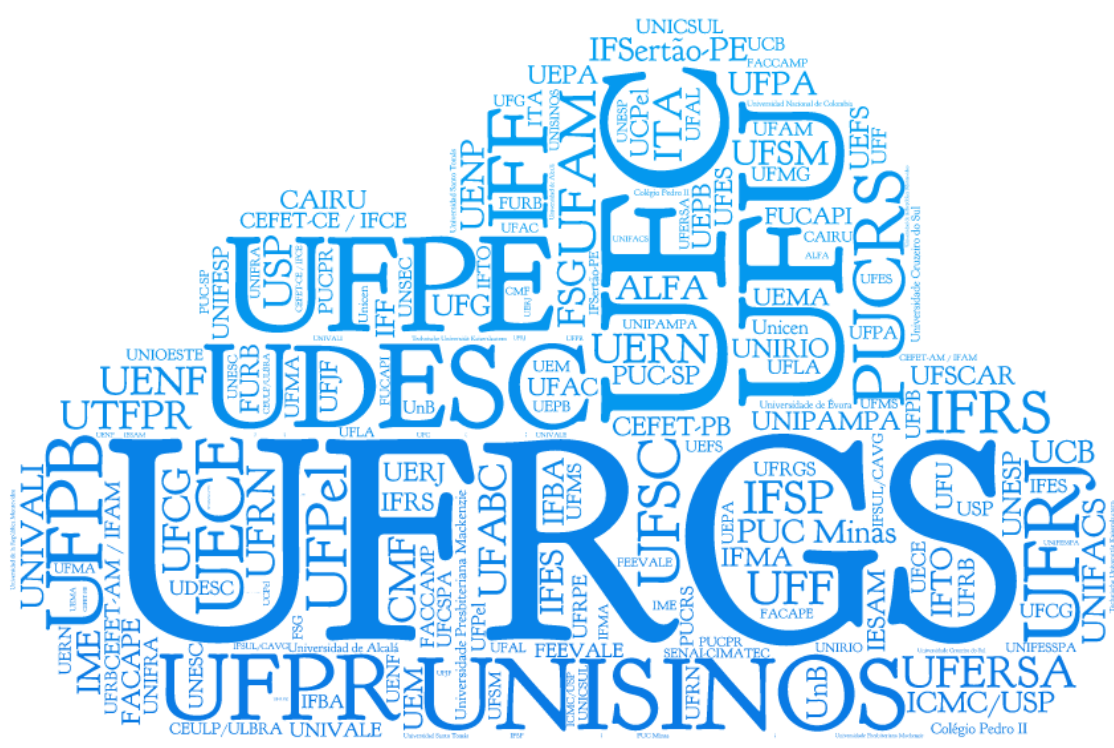

Figura 5. Nuvem de tags das principais Universidades. 
V Congresso Brasileiro de Informática na Educação (CBIE 2016)

Anais do XXVII Simpósio Brasileiro de Informática na Educação (SBIE 2016)

A Figura 6 e a Figura 7 apresentam a representatividade dos estados brasileiros em relação à quantidade de artigos cadastrados. A Figura 7 destaca os dados em formato de coluna a representação de cada estado do Brasil e a Figura 8 apresenta o Mapa do Brasil com a respectiva representatividade de cada região do país. Vale mencionar que existem quatro autores estrangeiros que foram desconsiderados desta análise, de modo a considerar apenas os estados de coautores brasileiros dos referidos artigos.

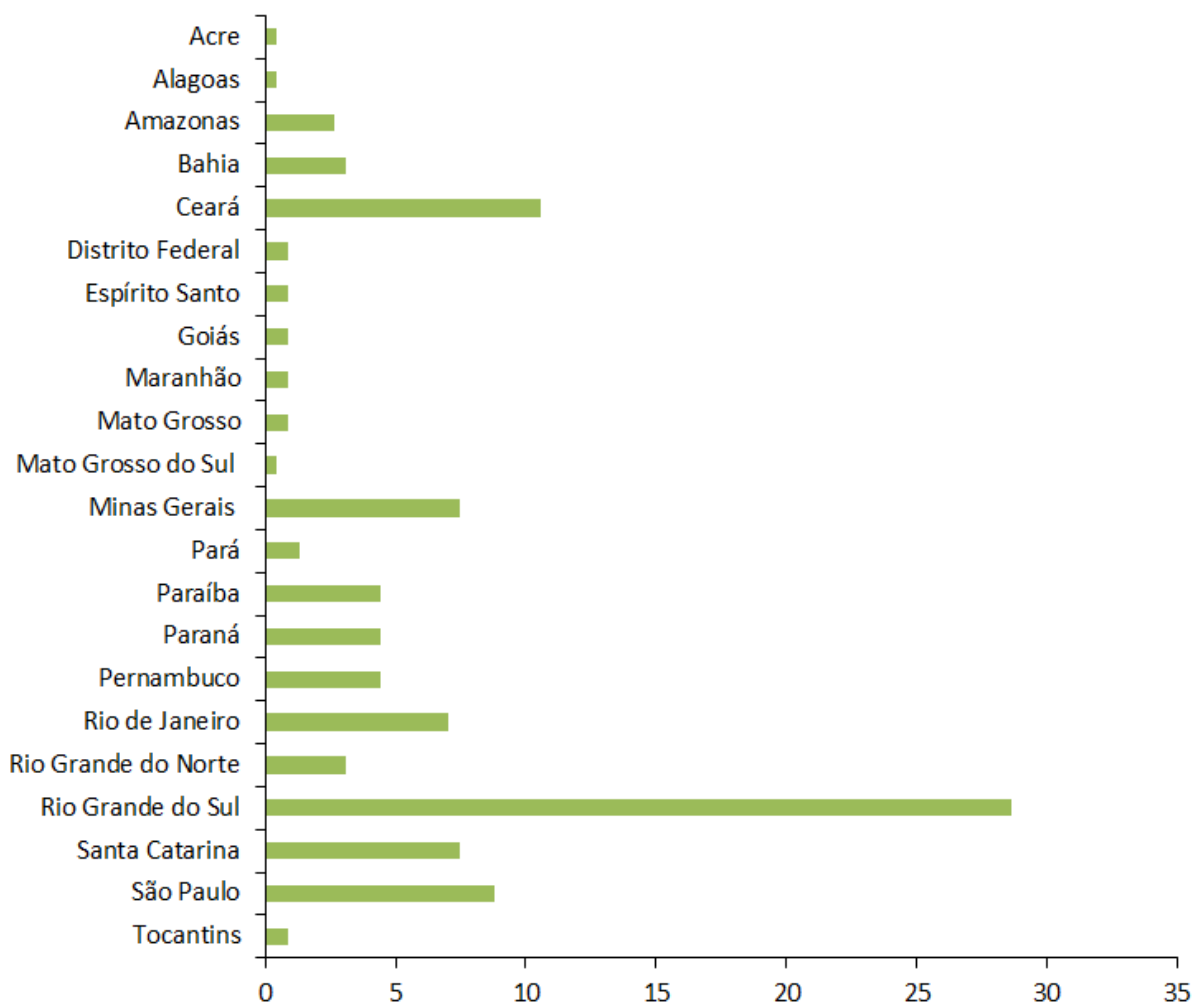

Figura 6. Representatividade de publicações por estado brasileiro.

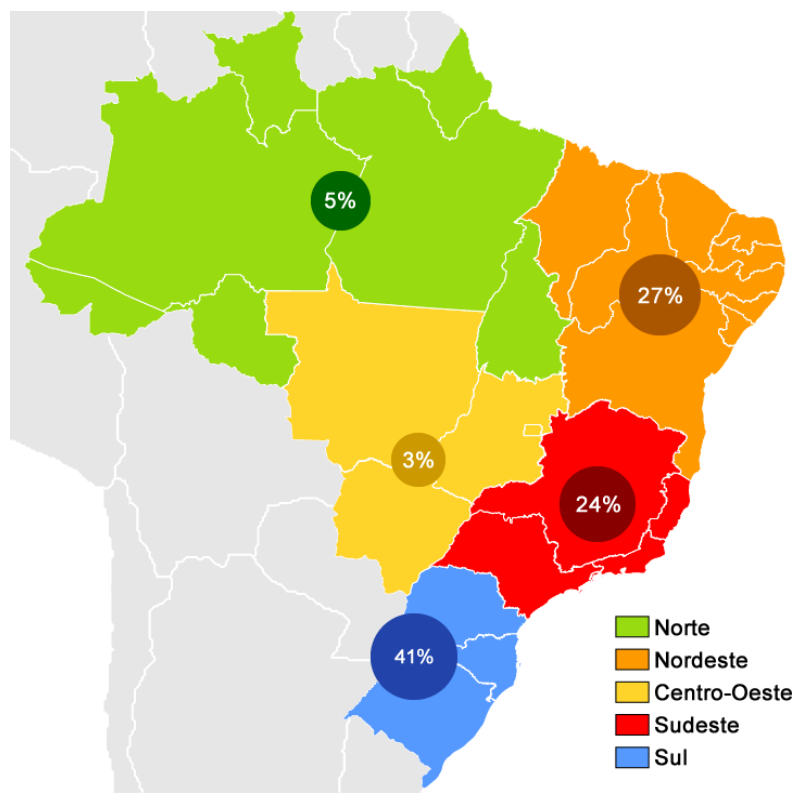

Figura 7. Representatividade de publicações por região brasileira. 
V Congresso Brasileiro de Informática na Educação (CBIE 2016)

Anais do XXVII Simpósio Brasileiro de Informática na Educação (SBIE 2016)

$\mathrm{Na}$ próxima seção, a análise é aprofundada com foco nas vertentes e nas iniciativas de pesquisa sobre objetos de aprendizagem no país.

\section{Vertentes e Iniciativas de Pesquisa}

Em busca de uma maior compreensão da pesquisa sobre objetos de aprendizagem no Brasil, nossa principal análise foi focada na categorização dos artigos. Sendo assim, de modo a classificar as publicações por assuntos, ou vertentes de pesquisa, chegou-se em doze categorias, em que os artigos foram classificados unitariamente em cada categoria, não havendo a possibilidade de uma publicação ser classificada por duas ou mais categorias. A Figura 8 ilustra as categorias elaboradas e sua representatividade do total de publicações analisadas.

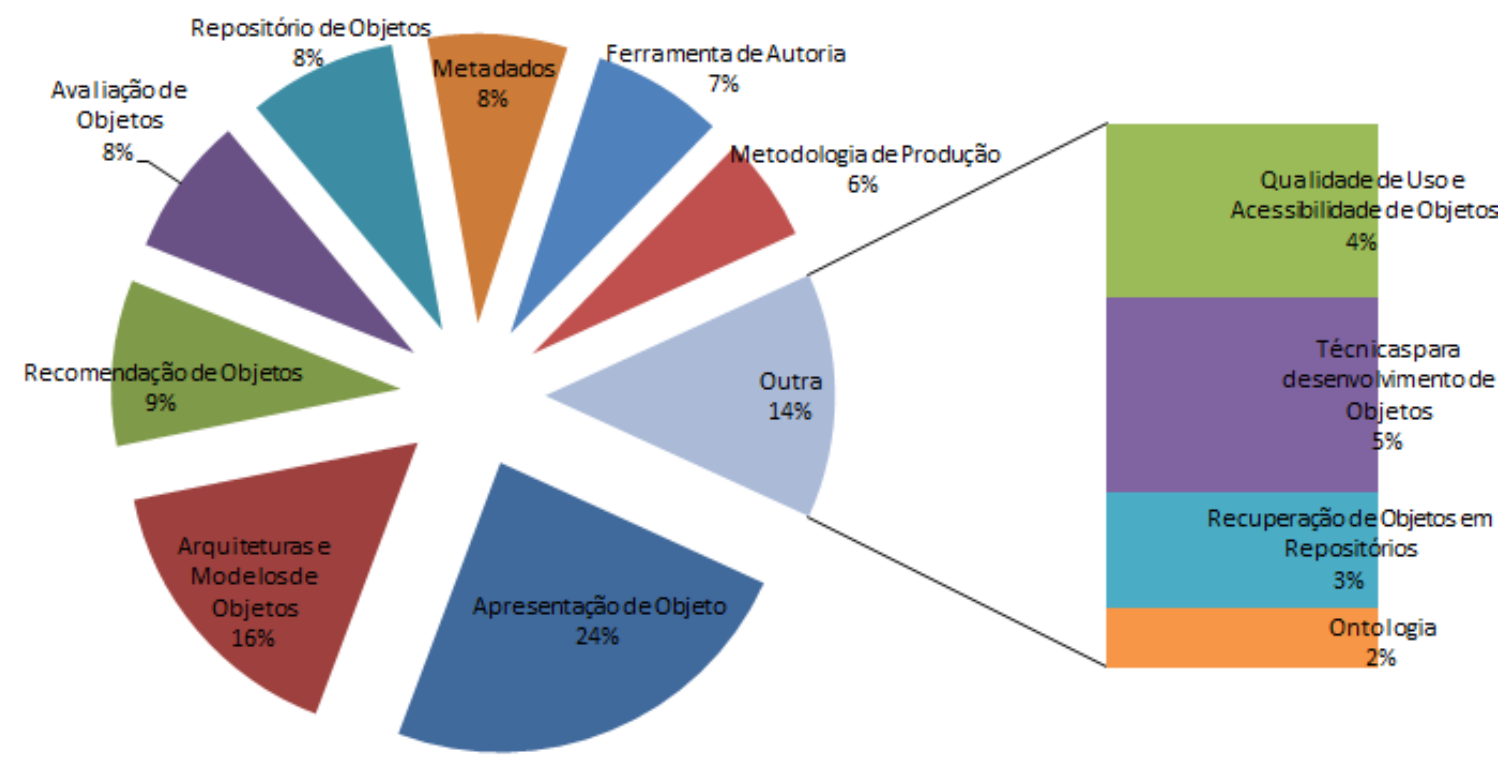

Figura 8. Representatividade de cada categoria do total de artigos.

Essas categorias, surgidas a partir da leitura e primeira categorização ilustram um pouco do cenário de pesquisa de objetos de aprendizagem. A categoria mais representativa, "Apresentação de Objetos", por exemplo, busca por meio de um artigo, explicar a produção de um objeto específico, seus benefícios, e por vezes uma avaliação do objeto ou do seu uso por alunos, de modo a identificar vantagens, validar a usabilidade, acessibilidade, entre outros.

Atrelado à análise de categorias, buscou-se por meio de uma análise por linha do tempo, verificar quais eram as tendências dessas publicações durante o recorte de tempo adotado nesse mapeamento. A Figura 9 apresenta esse levantamento, dando destaque principalmente a "Apresentação de Objeto" nos anos de 2007 e 2015, "Avaliação de Objeto" no ano de 2008, "Metadados" em 2010, "Arquiteturas e Modelos de Objetos" nos anos de 2011 e 2012 e "Recomendação de Objetos" em 2013. 
V Congresso Brasileiro de Informática na Educação (CBIE 2016)

Anais do XXVII Simpósio Brasileiro de Informática na Educação (SBIE 2016)

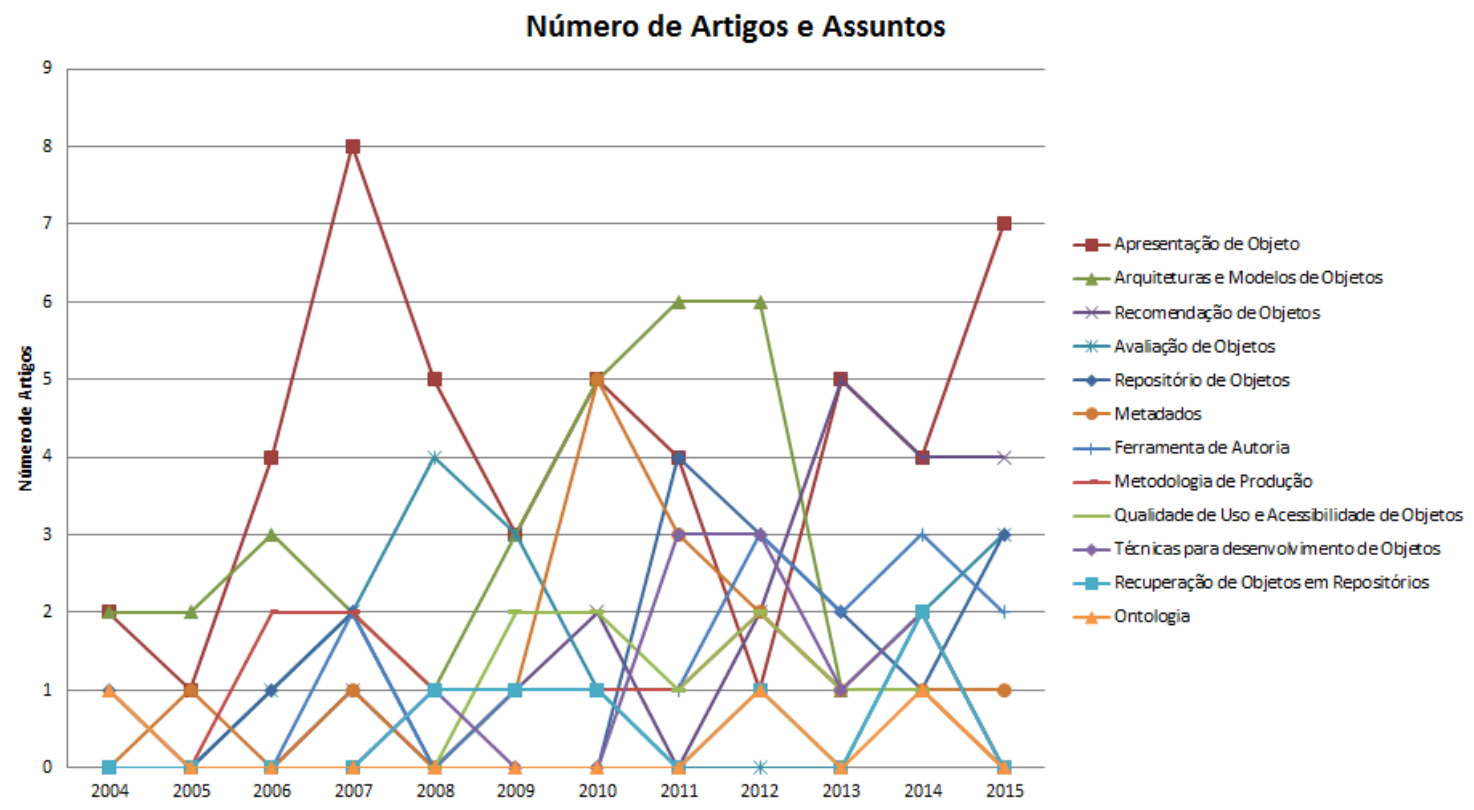

Figura 9. Número de artigos por categoria por ano.

Analisando o panorama geral das publicações, evidencia-se que - apesar da não continuidade de programas fomentadores da produção de objetos no país - a pesquisa sobre o tema continua estabelecida no Brasil, visto as potencialidades de uso destes recursos.

\section{Considerações Finais}

Neste artigo, apresentamos um panorama da pesquisa sobre objetos de aprendizagem por meio de um mapeamento sistemático a partir das publicações sobre o tema em artigos resumidos e completos de anais de eventos disponíveis digitalmente via CE-IE. Foram analisados os últimos 15 anos destas publicações relacionadas ao SBIE, WIE, DesafIE e workshops realizados no CBIE.

Por meio de diferentes formas de visualização, a partir da análise dessas publicações, esse estudo visa beneficiar principalmente pesquisadores que estejam iniciando seus estudos na área a compreender melhor o panorama de pesquisa atual no país, bem como demais pesquisadores, que por meio dela possam identificar principais autores e universidades envolvidas com pesquisas sobre o assunto, de modo a incentivar parcerias e o reuso de seus recursos educacionais.

Notoriamente estas considerações são preliminares, considerando que há muito mais análises que podem e devem ser realizadas a partir destes dados. Um dos principais trabalhos decorrentes desta pesquisa é a necessidade de expandir a pesquisa sobre o panorama brasileiro, incluindo a análise das publicações realizadas na Revista Brasileira de Informática na Educação (RBIE). Além disto, pretende-se explorar esses dados, investigando e mapeando os centros brasileiros de pesquisa e iniciativas de produção de objetos, de modo a compartilhar práticas e experiências, além de fomentar o reuso de objetos por meio de uma política de recursos educativos abertos.

Agradecimento: João Pedro Dewes Guterres agradece ao CNPq pela bolsa de estudos no Mestrado. 
V Congresso Brasileiro de Informática na Educação (CBIE 2016)

Anais do XXVII Simpósio Brasileiro de Informática na Educação (SBIE 2016)

\section{Referências}

Araújo, M.; Ferreira, M.A.G.V. (2004) "Construindo uma ontologia para pesquisa de Materiais e Objetos de Aprendizagem baseada na Web Semântica." In: XV SBIE (Simpósio Brasileiro de Informática na Educação), p. 369-378.

Bocchese, J.C.; Moraes, M.C.; Raymundo, V.P. (2013). Objeto pedagógico virtual sobre Processo de Coordenação. In: XIX WIE (Workshop de Informática na Escola), p. 150-159.

Brito, S.R.; Harb, M.P.A.A.; Favero, E.L.; Tavares, O.L. (2004) "Um sistema multiagente para o compartilhamento de objetos distribuídos de aprendizagem." In: XV SBIE (Simpósio Brasileiro de Informática na Educação), p. 399-408.

Carneiro, M.L.F.; Silveira, M.S. (2014) "Objetos de Aprendizagem como elementos facilitadores na Educação a Distância”. In: Educar em Revista, Curitiba, Brasil, Edição Especial n. 4, p. 235-260.

Cintra, V.P. (2010) "Projeto Rived: Um estudo de caso de uma equipe de matemática". Dissertação de Mestrado Educação Matemática, UNESP, 150p.

Creswell, J. W. (2013). "Research design: Qualitative, quantitative, and mixed methods approaches". Sage publications.

Damasceno, A.L.D.B.; Lima, T.S.; Neto, C.D.S.S. (2014). Cacuriá: Uma Ferramenta de Autoria Multimídia para Objetos de Aprendizagem. In Anais dos Workshops do Congresso Brasileiro de Informática na Educação (Vol. 3, No. 1, p. 76).

Gibbons, A. S.; Nelson J.; Richards, R. (2000) "The nature and origin of instructional objects". Disponível em: <http://reusability.org/read/chapters/gibbons.doc >. Acesso em 30 maio de 2016.

Gomes, E.R.; Silveira, R.A.; Vicari, R.M. (2004) "Objetos Inteligentes de Aprendizagem: uma abordagem baseada em agentes para objetos de aprendizagem". In: XV SBIE (Simpósio Brasileiro de Informática na Educação), p. 389-398.

Macêdo, G. T.; Gadelha, B.F., Mendonça, A.P., Martins, G.B., Junior, A.N.C. (2004) "Objetos de Aprendizagem: Uma Experiência de Integração com um Ambiente Telemático". In: XV SBIE (Simpósio Brasileiro de Informática na Educação), p. 379388 .

Moura, S.A.; Elia, M.F. (2004) "Projeto de recuperação paralela da Matemática Elementar baseado em Objetos de Aprendizagem Multimídia." In: XV SBIE (Simpósio Brasileiro de Informática na Educação), p. 35-37.

Pinho, M. S; Eliasquevici, M. K. "PitágorasNet: uma protótipo de objeto de aprendizagem para o ensino de Matemática". In: XIV Workshop de Informática na Escola (WIE), 2008.

Wiley, D. (2000) "Connecting learning objects to instructional design theory: a definition, a metaphor and a taxonomy". In: The instructional use of learning objects, 2000 [online]. Disponível em: <http://www.reusability.org/read/chapters/ wiley.doc $>$. Acesso em 30 maio de 2016. 